\title{
On 1/4 BPS ((F, D1), (NS5, D5)) bound states of type IIB string theory
}

\author{
Qiang Jia, ${ }^{a}$ J.X. Lu ${ }^{a}$ and Shibaji Roy ${ }^{b, c}$ \\ ${ }^{a}$ Interdisciplinary Center for Theoretical Study, University of Science and Technology of China, \\ Hefei, Anhui 230026, China \\ ${ }^{b}$ Saha Institute of Nuclear Physics, \\ 1/AF Bidhannagar, Calcutta-700 064, India \\ ${ }^{c}$ Homi Bhabha National Institute, \\ Training School Complex, Anushakti Nagar, Mumbai 400085, India \\ E-mail: skylo@mail.ustc.edu.cn, jxlu@ustc.edu.cn, \\ shibaji.roy@saha.ac.in
}

ABSTRACT: We construct two new $\mathrm{SL}(2, \mathbb{Z})$ invariant vacua of type IIB string theory which are bound states of $(p, q)$ strings with $(m, n)$ 5-branes, written as ((F, D1), (NS5, D5)) and preserve $1 / 4$ of the full space-time supersymmetries. For the first case, the strings live inside the 5-brane world-volume and in the second case the strings are perpendicular to the 5-brane world-volume. In the first case, naively one would expect an attractive interaction between the strings and the 5-branes due to attractive force between $\mathrm{F}$ and D5 and also between D1 and NS5. We find that 1/4 BPS bound state exists only when the vacuum moduli satisfy certain condition which is found to be consistent with the no-force condition between the branes. No such complication arises for the second case. The tension formulae and the various other descendant states which can be obtained by the application of T-duality for both these bound states are discussed.

KeYwords: p-branes, String Duality, Black Holes in String Theory

ARXIV EPRINT: 1704.01463 


\section{Contents}

1 Introduction 1

2 Type IIB supergravity and $\operatorname{SL}(2, \mathbb{R})$ symmetry 3

3 1/4 BPS ((F, D1), (NS5, D5)) from (D1, D5) 4

4 No-force condition $\quad 11$

5 Another 1/4 BPS ((F, D1), (NS5, D5)) from 1/4 BPS (F, D5) 14

$\begin{array}{llr}6 & \text { Conclusion } & 17\end{array}$

\section{Introduction}

Type IIB superstring theory in the low energy limit is well-known to possess a so-called Cremmer-Julia or classical U-duality $\mathrm{SL}(2, \mathbb{R})$ symmetry. Only the discrete subgroup, $\mathrm{SL}(2, \mathbb{Z})$ of this, is believed to survive quantum mechanically. Therefore, one would expect various vacuum-like BPS states of $\mathrm{SL}(2, \mathbb{Z})$ multiplets to exist in this theory. The classical U-duality group $\operatorname{SL}(2, \mathbb{R})$ is useful for the purpose of constructing such $\operatorname{SL}(2, \mathbb{Z})$ multiplets. It was Schwarz who first explicitly constructed the $\operatorname{SL}(2, \mathbb{Z})$ multiplet of $(p, q)$ strings in this theory [1]. Two of us then constructed the $\operatorname{SL}(2, \mathbb{Z})$ multiplet of $(m, n)$ 5-branes [2] and subsequently constructed the more complicated non-threshold bound state ((F, D1), (NS5, D5)), where, (NS5, D5) denotes the $(m, n) 5$-branes [3] and (F, D1) stands for the $(p, q)$ strings living along one of the $(m, n) 5$-brane spatial directions while delocalized along the other four.

However, all the $\operatorname{SL}(2, \mathbb{Z})$ multiplets mentioned so far are 1/2 BPS non-threshold bound states and their existence lends support to the conjectured quantum $\operatorname{SL}(2, \mathbb{Z})$ symmetry of type IIB superstring theory. For the $1 / 2$ BPS non-threshold bound state ((F, D1), (NS5, $\mathrm{D} 5))$, it was shown that the quantized charges for its $(p, q)$ strings and $(m, n) 5$-branes can not be completely arbitrary but must satisfy a relation given by, $(p, q)=k(a, b)$, $(m, n)=k^{\prime}(-b, a)$ with $(a, b)$ and $\left(k, k^{\prime}\right)$ being two pairs of co-prime integers. In particular, from this state we can recover $1 / 2$ BPS (F, D5) state by setting $a=1$ and $b=0$ and (D1, NS5) state by setting $a=0$ and $b=1$. We can also recover a delocalized $(p, q)$ string by putting $k^{\prime}=0$ as well as $(m, n) 5$-branes by putting $k=0$. However, because of the charge relation we just mentioned we can not recover either (D1, D5) or (F, NS5) bound state from the general state. This is entirely consistent with the fact that both these latter states are $1 / 4$ BPS threshold bound states and therefore should not be obtained from $1 / 2$ BPS non-threshold bound state ((F, D1), (NS5, D5)). Also it is known that both the $\mathrm{SL}(2, \mathbb{R})$ and the $\mathrm{SL}(2, \mathbb{Z})$ preserve the underlying supersymmetry of a given state on which they act. 
It is then natural to expect a new $1 / 4$ BPS $((\mathrm{F}, \mathrm{D} 1),(\mathrm{NS} 5, \mathrm{D} 5))$ bound state to exist given the existence of $1 / 4$ BPS (D1, D5) and (F, NS5). However, we know that there is no-force between D1 and D5 in the threshold bound state (D1, D5) (or between F and NS5 in (F, NS5)) and so if $1 / 4$ BPS $((F, D 1),(N S 5, D 5))$ bound state indeed exists, we expect that there should also be no-force between the non-threshold (F, D1) and the non-threshold (NS5, D5) and this bound state should also be threshold with respect to these two constituent non-threshold bound states. While it is true that there is no force between D1 and D5 and between F and NS5 in this bound state, we do have an attractive long-range force acting between F and D5 or between D1 and NS5 (for example, see [4]). We, therefore, expect a net attractive force between (F, D1) and (NS5, D5). This then appears to imply the existence of only the 1/2 BPS non-threshold ((F, D1), (NS5, D5)) bound state and not the 1/4 BPS threshold one, when (F, D1) is placed along one of the five world-volume spatial directions of (NS5, D5).

It would be very surprising if this turns out to be true because it would imply a potential problem with the conjectured quantum $\mathrm{SL}(2, \mathbb{Z})$ symmetry of Type IIB string theory given the existence of (D1, D5) and (F, NS5) quantum mechanically. ${ }^{1}$ In this paper, we will resolve this puzzle and find that there indeed exists a new type of ((F, D1), (NS5, D5)) bound state preserving $1 / 4$ of the spacetime supersymmetry and both (D1, D5) and (F, NS5) states can be obtained from it as special cases. Unlike the 1/2 BPS ((F, D1), (NS5, D5)) and also as anticipated, we expect certain constraint on the vacua, which manifest themselves in terms of the VEVs of the dilaton $\phi$ and RR 0 -form potential $\chi$, i.e., $\phi_{0}$ and $\chi_{0}$. It turns out that when $\phi_{0}$ and $\chi_{0}$ satisfy certain condition, the 1/4 BPS threshold ((F, D1), (NS5, D5)) bound state indeed exists. We also demonstrate that under precisely these conditions, there is no-force acting between (F, D1) and (NS5, D5) in this threshold bound state, lending support to the existence of this state.

The main purpose of the present paper is to address the aforementioned puzzle and to construct such a state in type IIB string theory. To construct this state we need to begin with a known threshold 1/4 BPS (D1, D5) state or (F, NS5) state and apply $\operatorname{SL}(2, \mathbb{R})$ transformation and the charge quantizations of the various constituent branes. We will also spell out some peculiarities in the construction not encountered before in similar constructions. Also for completeness we will also construct another $1 / 4$ BPS bound state of the same form but here (F, D1) strings will not be along (NS5, D5) brane spatial directions, but will be perpendicular to them. In this case, we will start with the known $1 / 4$ BPS (F, D5) bound state solution of type IIB string theory and apply an $\operatorname{SL}(2, \mathbb{R})$ rotation and charge quantization to it. The charge relation in this case will be very similar to that of the $1 / 2$ BPS ((F, D1), (NS5, D5)) solution we constructed before [3]. This will also serve as a contrast between these two kinds of $1 / 4$ BPS states.

This paper is organized as follows. In the next section we briefly discuss $\operatorname{SL}(2, \mathbb{R})$ symmetry of low energy type IIB string theory and fix our notations and conventions. In section 3 , we construct $\mathrm{SL}(2, \mathbb{Z})$ multiplet of $1 / 4$ BPS ((F, D1), (NS5, D5)) solution

\footnotetext{
${ }^{1}$ The low energy classical U-duality SL $(2, \mathbb{R})$ symmetry can always be used to generate the classical solution 1/4 BPS ((F, D1), (NS5, D5)) from the given classical 1/4 BPS (D1, D5) or (F, NS5).
} 
starting from (D1, D5) solution using $\mathrm{SL}(2, \mathbb{R})$ and then imposing charge quantizations of constituent branes. We also derive the condition for the existence of such a state. In section 4, we consider a probe (F, D1)-string placed in (NS5, D5) brane background and derive the no-force condition. It turns out that the no-force condition is precisely the one derived in section 3. In section 5, we construct the other $1 / 4$ BPS ((F, D1), (NS5, D5)) solution starting from 1/4 BPS (F, D5) solution. Our conclusion is given in section 5.

\section{Type IIB supergravity and $\mathrm{SL}(2, \mathbb{R})$ symmetry}

In this section we briefly review the low energy effective Lagrangian of type IIB string theory (type IIB supergravity [5]) and mention how it can be written in an $\operatorname{SL}(2, \mathbb{R})$ invariant form. This will also fix our notations and conventions. The bosonic part of the Lagrangian has the form (see for example, [6]),

$$
\begin{aligned}
\mathcal{L}_{\mathrm{IIB}}= & R * 1-\frac{1}{2} * d \phi \wedge d \phi-\frac{1}{2} e^{2 \phi} * d \chi \wedge d \chi-\frac{1}{4} * H_{5} \wedge H_{5} \\
& -\frac{1}{2} e^{-\phi} * F_{3}^{\mathrm{NS}} \wedge F_{3}^{\mathrm{NS}}-\frac{1}{2} e^{\phi} * \tilde{F}_{3} \wedge \tilde{F}_{3}-\frac{1}{2} B_{4} \wedge F_{3}^{R R} \wedge F_{3}^{\mathrm{NS}},
\end{aligned}
$$

where $* \mathbf{1}$ stands for the 10 dimensional volume-form, $R$, the Ricci scalar, $\phi$, the dilaton, $F_{3}^{\mathrm{NS}}$, the NSNS 3-form field strength, $\chi$, the RR 0 -form potential, $F_{3}^{\mathrm{RR}}$, the RR 3 -form field strength and $B_{4}$, the RR 4 -form potential. In the above $*$ denotes the spacetime Hodge-dual, and

$$
\begin{aligned}
F_{3}^{\mathrm{NS}} & =d A_{2}^{\mathrm{NS}}, \quad \tilde{F}_{3}=d A_{2}^{\mathrm{RR}}+\chi F_{3}^{\mathrm{NS}}, \\
H_{5} & =d B_{4}-\frac{1}{2} A_{2}^{\mathrm{RR}} \wedge F_{3}^{\mathrm{NS}}+\frac{1}{2} A_{2}^{\mathrm{NS}} \wedge F_{3}^{\mathrm{RR}} .
\end{aligned}
$$

In eq. (2.1), we have included $H_{5}$ in the Lagrangian for the purpose of equations of motion for the other fields and at the end its self-dual relation $* H_{5}=H_{5}$ is imposed by hand.

From the above Lagrangian, we have the following equations of motion

$$
\begin{aligned}
R_{M N}= & \frac{1}{2} \partial_{M} \phi \partial_{N} \phi+\frac{1}{2} e^{2 \phi} \partial_{M} \chi \partial_{N} \chi+\frac{1}{96} H_{M N}^{2} \\
& +\frac{1}{4} e^{\phi}\left[\left(\tilde{F}_{3}\right)_{M N}^{2}-\frac{1}{12} g_{M N}\left(\tilde{F}_{3}\right)^{2}\right] \\
& +\frac{1}{4} e^{-\phi}\left[\left(F_{3}^{\mathrm{NS}}\right)_{M N}^{2}-\frac{1}{12} g_{M N}\left(F_{3}^{\mathrm{NS}}\right)^{2}\right], \\
d * d \phi= & -e^{2 \phi} * d \chi \wedge d \chi-\frac{1}{2} e^{\phi} * \tilde{F}_{3} \wedge \tilde{F}_{3}+\frac{1}{2} e^{-\phi} * F_{3}^{\mathrm{NS}} \wedge F_{3}^{\mathrm{NS}}, \\
d\left(e^{2 \phi} * d \chi\right)= & -e^{\phi} * \tilde{F}_{3} \wedge F_{3}^{\mathrm{NS}}, \quad d * H_{5}=-F_{3}^{\mathrm{RR}} \wedge F_{3}^{\mathrm{NS}}\left(\text { with } * H_{5}=H_{5}\right), \\
d\left(e^{\phi} * \tilde{F}_{3}\right)= & H_{5} \wedge F_{3}^{\mathrm{NS}}, \quad d\left(e^{-\phi} * F_{3}^{\mathrm{NS}}+\chi e^{\phi} * \tilde{F}_{3}\right)=-H_{5} \wedge F_{3}^{\mathrm{RR}} .
\end{aligned}
$$

In order to see the manifest $\operatorname{SL}(2, \mathbb{R})$ symmetry, we now try to re-express the Lagrangian (2.1) and the fields $(2.2)$ in an $\operatorname{SL}(2, \mathbb{R})$ invariant or covariant form. Note 
that the Einstein frame metric and the RR 5 -form are invariant under $\mathrm{SL}(2, \mathbb{R})$. The totally antisymmetric tensor $\epsilon^{i j}$, where $i, j=1,2$ and $\epsilon^{12}=1$, is $\operatorname{SL}(2, \mathbb{R})$ invariant. As usual, we define $F_{3}^{(1)}=F_{3}^{\mathrm{NS}}$ and $F_{3}^{(2)}=F_{3}^{\mathrm{RR}}$. We also define a 3 -form vector $F_{3}$ and a $2 \times 2$ scalar matrix $\mathcal{M}$ as

$$
F_{3}=\left(\begin{array}{c}
F_{3}^{(1)} \\
F_{3}^{(2)}
\end{array}\right), \quad \mathcal{M}=\left(\begin{array}{cc}
\chi^{2}+e^{-2 \phi} & \chi \\
\chi & 1
\end{array}\right) e^{\phi} .
$$

With the above, the Lagrangian can be re-expressed as

$$
\begin{aligned}
\mathcal{L}_{\mathrm{IIB}}= & R * \mathbf{1}-\frac{1}{4} * H_{5} \wedge H_{5}+\frac{1}{4} T r * d \mathcal{M} \wedge d \mathcal{M}^{-1}-\frac{1}{2} * F_{3}^{T} \wedge \mathcal{M} F_{3} \\
& +\frac{1}{4} \epsilon^{i j} B_{4} \wedge F_{3}^{(i)} \wedge F_{3}^{(j)} .
\end{aligned}
$$

This is manifestly $\mathrm{SL}(2, \mathbb{R})$ invariant if we have

$$
\mathcal{M} \rightarrow \Lambda \mathcal{M} \Lambda^{T}, \quad F_{3} \rightarrow\left(\Lambda^{-1}\right)^{T} F_{3}, \quad g_{M N} \rightarrow g_{M N}
$$

where

$$
\Lambda^{i l} \Lambda^{j k} \epsilon^{l k}=\epsilon^{i j},
$$

defines $\Lambda$ as a $2 \times 2 \mathrm{SL}(2, \mathbb{R})$ matrix. In general, we can have

$$
\Lambda=\left(\begin{array}{ll}
\alpha & \beta \\
\gamma & \delta
\end{array}\right), \quad \text { with } \quad \alpha \delta-\beta \gamma=1 .
$$

If we express the dilaton $\phi$ and the RR 0 -form potential $\chi$ in terms of $\lambda=\chi+i e^{-\phi}$, it will transform fractional linearly under $\operatorname{SL}(2, \mathbb{R})$ as

$$
\lambda \rightarrow \lambda^{\prime}=\frac{\alpha \lambda+\beta}{\gamma \lambda+\delta}
$$

The $H_{5}$ in $(2.2)$ can now be written as,

$$
H_{5}=d B_{4}+\frac{1}{2} \epsilon^{i j} A_{2}^{(i)} \wedge F_{3}^{(j)}, \quad d * H_{5}=\frac{1}{2} \epsilon^{i j} F_{3}^{(i)} \wedge F_{3}^{(j)},
$$

which is also $\operatorname{SL}(2, \mathbb{R})$ invariant as expected.

\section{1/4 BPS ((F, D1), (NS5, D5)) from (D1, D5)}

In this section we will construct an $\mathrm{SL}(2, \mathbb{Z})$ multiplet of states in the form of $1 / 4$ BPS threshold bound states ((F, D1), (NS5, D5)) starting from the well-known 1/4 BPS threshold classical solution (D1, D5) of type IIB string theory by an $\mathrm{SL}(2, \mathbb{R})$ transformation and 
then imposing the charge quantizations of the different constituent branes. The field configuration for the (D1, D5) state (for example, see [7]) is,

$$
\begin{aligned}
d s^{2}= & H_{1}^{-3 / 4} H_{5}^{-1 / 4}\left(-d t^{2}+\left(d x^{5}\right)^{2}\right)+H_{1}^{1 / 4} H_{5}^{-1 / 4} \sum_{i=1}^{4}\left(d x^{i}\right)^{2} \\
& +H_{1}^{1 / 4} H_{5}^{3 / 4}\left(d r^{2}+r^{2} d \Omega_{3}^{2}\right), \\
e^{2 \phi}= & H_{1} / H_{5}, \\
F_{3}^{(2)}= & \frac{2 Q_{1}}{H_{1}^{2} r^{3}} d t \wedge d x^{5} \wedge d r+2 Q_{5} \epsilon_{3},
\end{aligned}
$$

where the two harmonic functions $H_{1 / 5}=1+Q_{1 / 5} / r^{2}, \epsilon_{3}$ is the volume 3 -form of a unit 3 -sphere and parameters $Q_{1}, Q_{5}$ are related to the D1 and D5 charges as

$$
e_{1}=\frac{2 Q_{1} \Omega_{3} V_{4}}{\sqrt{2} \kappa_{0}}, \quad g_{5}=\frac{2 Q_{5} \Omega_{3}}{\sqrt{2} \kappa_{0}},
$$

with $\Omega_{3}=2 \pi^{2}$ the volume of unit 3 -sphere, $\sqrt{2} \kappa_{0}=(2 \pi)^{7 / 2} \alpha^{\prime 2}$ and $V_{4}=\int d x^{1} \wedge d x^{2} \wedge d x^{3} \wedge d x^{4}$. The D-strings are along $x^{5}$ direction but delocalized along 1,2,3,4-directions while the D5-branes are along $x^{1}, x^{2}, \cdots, x^{5}$ directions. In the above, we have set the asymptotic value $\phi_{0}=0, \chi_{0}=0$.

The equation of motion for $A_{2}^{(i)}$ can now be obtained from the $\operatorname{SL}(2, \mathbb{R})$ invariant Lagrangian (2.5) as

$$
d\left(\mathcal{M}^{i j} * F_{3}^{(j)}\right)=-\epsilon^{i j} H_{5} \wedge F_{3}^{(j)}
$$

where we have used (2.10) and $* H_{5}=H_{5}$. This one agrees with the two equations in the last line of (2.3). This equation can also be re-written as

$$
d\left(\mathcal{M}^{i j} * F_{3}^{(j)}+\epsilon^{i j} B_{4} \wedge F_{3}^{(j)}-\frac{1}{2} A_{2}^{(i)} \wedge A_{2}^{(j)} \wedge F_{3}^{(j)}\right)=0
$$

from which we can define the electric-like (F, D1) string charge $e_{1}^{T}=\left(e_{1}^{(1)}, e_{1}^{(2)}\right)$ as

$$
e_{1}^{(i)}=\frac{1}{\sqrt{2} \kappa_{0}} \int_{R^{4} \times S_{\infty}^{3}} \mathcal{M}^{i j} * F_{3}^{(j)}+\epsilon^{i j} B_{4} \wedge F_{3}^{(j)}-\frac{1}{2} A_{2}^{(i)} \wedge A_{2}^{(j)} \wedge F_{3}^{(j)},
$$

while the magnetic-like (NS5, D5) brane charge $g_{5}^{T}=\left(g_{5}^{(1)}, g_{5}^{(2)}\right)$ is defined as

$$
g_{5}^{(i)}=\frac{1}{\sqrt{2} \kappa_{0}} \int_{S_{\infty}^{3}} F_{3}^{(i)} .
$$

For the present case, the Chern-Simons terms in the electric charge never contribute and so we drop them from now on. Given the $\operatorname{SL}(2, \mathbb{R})$ transformations of $\mathcal{M}$ and $F$ in (2.6), we have the electric-like charge $e_{1}$ and magnetic-like charge $g_{5}$ to transform as

$$
e_{1} \rightarrow \Lambda e_{1}, \quad g_{5} \rightarrow\left(\Lambda^{-1}\right)^{T} g_{5} .
$$

The standard choice for $\Lambda$ is

$$
\Lambda=\left(\begin{array}{cc}
e^{-\phi_{0}} \cos \alpha+\chi_{0} \sin \alpha-e^{-\phi_{0}} \sin \alpha+\chi_{0} \cos \alpha \\
\sin \alpha & \cos \alpha
\end{array}\right) e^{\phi_{0} / 2} .
$$


Since (D1, D5) is our initial classical configuration with classical D1 charge $\Delta_{1}^{1 / 2}$ and classical D5 charge $\Delta_{5}^{1 / 2}$, respectively, we have

$$
e_{1}=\left(\begin{array}{c}
0 \\
\Delta_{1}^{1 / 2}
\end{array}\right) e_{10}, \quad g_{5}=\left(\begin{array}{c}
0 \\
\Delta_{5}^{1 / 2}
\end{array}\right) g_{50},
$$

where $e_{10}$ and $g_{50}$ are the unit charges for strings and 5-branes, respectively. After the $\mathrm{SL}(2, \mathbb{R})$ transformation, we should have the transformed classical charges and after imposing their respective quantizations, we end up with $\bar{e}_{1}^{T}=(p, q) e_{10}$, and $\bar{g}_{5}^{T}=(m, n) g_{50}$ with $(p, q)$ and $(m, n)$ each being a pair of integers. Using these quantization conditions we get,

$$
\begin{aligned}
& \cos \alpha=q e^{-\phi_{0} / 2} \Delta_{1}^{-1 / 2}=\left(m \chi_{0}+n\right) e^{\phi_{0} / 2} \Delta_{5}^{-1 / 2}, \\
& \sin \alpha=\left(q \chi_{0}-p\right) e^{\phi_{0} / 2} \Delta_{1}^{-1 / 2}=-m e^{-\phi_{0} / 2} \Delta_{5}^{-1 / 2} .
\end{aligned}
$$

Using (3.10) we get,

$$
\begin{aligned}
\Delta_{1} & =q^{2} e^{-\phi_{0}}+\left(q \chi_{0}-p\right)^{2} e^{\phi_{0}}, \quad \Delta_{5}=m^{2} e^{-\phi_{0}}+\left(m \chi_{0}+n\right)^{2} e^{\phi_{0}}, \\
\tan \alpha & =\frac{\left(q \chi_{0}-p\right)}{q} e^{\phi_{0}}=-\frac{m}{\left(m \chi_{0}+n\right)} e^{-\phi_{0}},
\end{aligned}
$$

and from the last relation we have,

$$
\left(m \chi_{0}+n\right)\left(q \chi_{0}-p\right)=-m q e^{-2 \phi_{0}}
$$

which can actually be expressed as an $\mathrm{SL}(2, \mathbb{Z})$ invariant form ${ }^{2}$

$$
(p, q) \epsilon^{T} \mathcal{M}_{0}\left(\begin{array}{c}
m \\
n
\end{array}\right)=0
$$

where $\epsilon$ denotes the $\mathrm{SL}(2, \mathbb{Z})$ invariant antisymmetric matrix ${ }^{3}$ defined in (2.7). Eq. (3.12) can be satisfied with arbitrary $\phi_{0}$ and $\chi_{0}$, only if $q m=0, p n=0$ and also $(q n-p m)=0$. However, we note that these relations are inconsistent even for the (D1, D5) configuration ${ }^{4}$ for which $q$ and $n$ are both non-zero. Thus we conclude that for $\operatorname{SL}(2, \mathbb{Z})$ multiplet of $1 / 4$ BPS ((F, D1), (NS5, D5)) bound state to exist, $\phi_{0}$ and/or $\chi_{0}$ can not be arbitrary but must take specific values such that they satisfy the condition (3.12). We would like to emphasize that in this bound state $(p, q)$ strings $(\mathrm{F}, \mathrm{D} 1)$ form a threshold bound state with $(m, n)$ 5 -branes (NS5, D5) which is implied from the original (D1, D5) metric since the total energy is simply the sum of those of the delocalized (F, D1) and (NS5, D5). Therefore, there is no interaction between (F, D1) and (NS5, D5) just as there is no interaction between the original D1 and D5. This is far from obvious since in general we do expect interaction

\footnotetext{
${ }^{2}$ We thank one of our referees for pointing this out to us.

${ }^{3}$ Note also $\Lambda^{T} \epsilon^{T} \Lambda=\epsilon^{T}$ in addition to $\Lambda \epsilon \Lambda^{T}=\epsilon$ defined in (2.7).

${ }^{4}$ Even though we have chosen the initial (D1, D5) configuration (3.1) with $\phi_{0}=\chi_{0}=0$, it can have arbitrary $\phi_{0}$ and constant $\chi_{0}$ as can be seen from the corresponding equations of motion (2.3), even after imposing the charge quantizations of D1 and D5, respectively.
} 
between (F, D1) and (NS5, D5) since we have attractive interaction between F and D5 and also between D1 and NS5 [4]. We will demonstrate explicitly in the following section by computing the force experienced by the $(p, q)$ string in the $(m, n) 5$-brane background that indeed the no-force condition is precisely the same as the condition (3.12).

One important point to note about (3.12) is that, the quantized charges of various objects in this bound state appear to get fixed by the moduli $\phi_{0}$ and $\chi_{0}$. But that is not desirable and not right since the values of the moduli are given and the quantization condition is imposed later and therefore charges should be able to take any quantized value independent of $\phi_{0}$ and $\chi_{0}$. In order to achieve this we re-write $(p, q)=k(a, b)$ and $(m, n)=k^{\prime}\left(a^{\prime}, b^{\prime}\right)$, where $(a, b),\left(a^{\prime}, b^{\prime}\right)$ are two pairs of co-prime integers and $k, k^{\prime}$ are integers but not necessarily co-prime. This is because (F, D1) and (NS5, D5) form only threshold bound state just like original (D1, D5). For the non-degenerate configuration $k k^{\prime} \neq 0$ and so, substituting these values of $(p, q)$ and $(m, n)$ in (3.12), we find that $\phi_{0}$ and $\chi_{0}$ are not linked with $(p, q)$ and $(m, n)$, but rather with $(a, b)$ and $\left(a^{\prime}, b^{\prime}\right)$. Actually the charges $(p, q)$ and $(m, n)$ can take almost any arbitrary integer values except for the degenerate $k k^{\prime}=0$ cases.

For the concerned state to remain $1 / 4$ BPS, we must have $k k^{\prime} \neq 0$. The degenerate $k \neq 0, k^{\prime}=0$ or $k=0, k^{\prime} \neq 0$ case implies that the underlying state is either delocalized $1 / 2$ BPS $(p, q)$-strings or 1/2 BPS $(m, n) 5$-branes. However, for either of these two cases, both (3.10) and (3.11) are not well-defined and for this reason we don't expect (3.12) to hold good. This is entirely consistent since (3.12) is the constraint for $1 / 4$ BPS state, not for $1 / 2$ BPS state. The condition $k k^{\prime} \neq 0$ implies that none of the two integers in either $(p, q)$ or $(m, n)$ pair can vanish. We remark here that (3.12) rules out the existence of (F, D5) or (D1, NS5). For the former, we have $p, n \neq 0, q=m=0$ and (3.12) gives $p n=0$, contradicting our assumption, while for the latter, we have $q, m \neq 0, p=n=0$ and (3.12) gives $e^{-2 \phi_{0}}=-\chi_{0}^{2}$, impossible to hold. This is also consistent since either of these two is a $1 / 2$ BPS state. To make our following discussion definite, we are assuming $k k^{\prime} \neq 0$ from now on. Then $(3.12)$ becomes, in terms of $(a, b)$ and $\left(a^{\prime}, b^{\prime}\right)$, as

$$
\left(a^{\prime} \chi_{0}+b^{\prime}\right)\left(b \chi_{0}-a\right)=-a^{\prime} b e^{-2 \phi_{0}} .
$$

We also have now

$$
\begin{aligned}
& \cos \alpha=b e^{-\phi_{0} / 2} \tilde{\Delta}_{1}^{-1 / 2}=\left(a^{\prime} \chi_{0}+b^{\prime}\right) e^{\phi_{0} / 2} \tilde{\Delta}_{5}^{-1 / 2}, \\
& \sin \alpha=\left(b \chi_{0}-a\right) e^{\phi_{0} / 2} \tilde{\Delta}_{1}^{-1 / 2}=-a^{\prime} e^{-\phi_{0} / 2} \tilde{\Delta}_{5}^{-1 / 2},
\end{aligned}
$$

where

$$
\tilde{\Delta}_{1}=b^{2} e^{-\phi_{0}}+\left(b \chi_{0}-a\right)^{2} e^{\phi_{0}}, \quad \tilde{\Delta}_{5}=a^{\prime 2} e^{-\phi_{0}}+\left(a^{\prime} \chi_{0}+b^{\prime}\right)^{2} e^{\phi_{0}} .
$$

We would like to address first a few subtleties regarding the generation of (D1, D5) (or $(\mathrm{F}, \mathrm{NS} 5))$ solution with non-zero moduli $\left(\chi_{0}, \phi_{0} \neq 0\right)$ from the same solution with zero moduli $\left(\chi_{0}=\phi_{0}=0\right)$ before we proceed to discuss the general 1/4 BPS state including certain special cases using (3.14). We note that the $\operatorname{SL}(2, \mathbb{R})$ matrix given in (3.8) can be 
decomposed as,

$$
\Lambda=\left(\begin{array}{cc}
e^{-\phi_{0}} & \chi_{0} \\
0 & 1
\end{array}\right) e^{\phi_{0} / 2}\left(\begin{array}{cc}
\cos \alpha & -\sin \alpha \\
\sin \alpha & \cos \alpha
\end{array}\right) \equiv \Lambda_{0} \Lambda_{R}
$$

where $\Lambda_{R}$ is the $\mathrm{SO}(2)$ rotation subgroup of $\mathrm{SL}(2, \mathbb{R}) . \Lambda_{R}$ actually generates new solutions, i.e., starting from (D1, D5) with $\phi_{0}=\chi_{0}=0$ it generates another solution with again zero moduli since $\Lambda_{R} \mathbf{I} \Lambda_{R}^{T}=\mathbf{I}$. On the other hand, $\Lambda_{0}$ generates (D1, D5) solution with non-zero moduli $\left(\chi_{0}, \phi_{0} \neq 0\right)$ from the same solution with zero moduli $\left(\chi_{0}=\phi_{0}=0\right)$. The last statement can be justified by looking at the relation $\mathcal{M}_{0}=\Lambda_{0} \mathbf{I} \Lambda_{0}^{T}$, where $\mathcal{M}_{0}$ is the matrix given in (2.4) with the asymptotic values of the moduli and $\mathbf{I}$ is the $2 \times 2$ identity matrix which is nothing but $\mathcal{M}_{0}$ with the moduli put to zero. Since there are no F-strings and/or NS5-branes generated under the special $\mathrm{SL}(2, \mathbb{R})$ transformation of $\Lambda=\Lambda_{0}$, we should not blindly enforce the charge quantization for either of these two kinds of branes. In other words, we don't have (3.10) and (3.11) for this case and so (3.12) also does not hold. So $\phi_{0}$ and $\chi_{0}$ can be arbitrary for (D1, D5), not constrained by (3.12). This same discussion holds true if we replace (D1, D5) by (F, NS5) in the above. In general, (D1, D5) (or (F, NS5)) are physically different with different $\phi_{0}, \chi_{0}$ except for the special case $\phi_{0}=0, \chi_{0}=$ interger. This latter special case is physically equivalent to (D1, D5) (or (F, NS5)) with $\phi_{0}=\chi_{0}=0$ since these two are related by an $\operatorname{SL}(2, \mathbb{Z})$ transformation $\Lambda_{0}$ with $\phi_{0}=0, \chi_{0}=$ integer.

We now come to give a general discussion on the non-degenerate 1/4 BPS threshold ((F, D1), (NS5, D5)) state including certain special cases using (3.14) as well as (3.15). Since the pair $(a, b)$ (or $\left.\left(a^{\prime}, b^{\prime}\right)\right)$ are co-prime, so at most one of two co-prime integers can vanish. We will discuss case by case in the following.

Case $1 \boldsymbol{a}=\mathbf{0 ,} \boldsymbol{b} \neq \mathbf{0}$. For this, from (3.14), we have $a^{\prime} e^{-2 \phi_{0}}=-\chi_{0}\left(a^{\prime} \chi_{0}+b^{\prime}\right)$. We have three subcases to consider: 1a) $a^{\prime}=0, b^{\prime} \neq 0$, then we must have $\chi_{0}=0$ with $\phi_{0}$ arbitrary. This subcase is just $1 / 4$ BPS (D1, D5) with $\chi_{0}=0$ and arbitrary $\phi_{0}$. For $1 / 4$ BPS (D1, D5), our previous discussion says that it can exist with arbitrary $\chi_{0}$ and $\phi_{0}$. The restriction $\chi_{0}=0$ obtained here is due to the charge quantization condition imposed for F-string and NS5-brane when we consider 1/4 BPS ((F, D1), (NS5, D5)). However, this condition is actually irrelevant when we merely consider (D1, D5) and should be dropped. 1b) $a^{\prime} \neq 0, b^{\prime}=0$. This subcase is impossible since it requires $e^{-2 \phi_{0}}=-\chi_{0}^{2}$. This just tells us that $1 / 4$ BPS (D1, NS5) does not exist as we discussed previously. 1c) $a^{\prime} \neq 0, b^{\prime} \neq 0$. Now we have $e^{-2 \phi_{0}}=-\chi_{0}\left(\chi_{0}+b^{\prime} / a^{\prime}\right)$, which gives a restriction on $\chi_{0}$ as $-b^{\prime} / a^{\prime}<\chi_{0}<0$ if $a^{\prime} b^{\prime}>0$ or $0<\chi_{0}<-b^{\prime} / a^{\prime}$ if $a^{\prime} b^{\prime}<0$. This subcase gives $1 / 4$ BPS (D1, (NS5, D5)) if the moduli $\phi_{0}$ and $\chi_{0}$ satisfy these constraints.

Case $2 \boldsymbol{a} \neq \mathbf{0}, \boldsymbol{b}=\mathbf{0}$. For this, we have $a^{\prime} \chi_{0}+b^{\prime}=0$ from (3.14). So $a^{\prime}$ cannot be zero and we have two subcases to consider. 2a) $a^{\prime} \neq 0, b^{\prime}=0$. This gives $\chi_{0}=0$. We have now $1 / 4$ BPS (F, NS5) with $\chi_{0}=0$ and arbitrary $\phi_{0}$. By the same argument discussed in subcase 1a) for (D1, D5), this case actually works for arbitrary $\chi_{0}$ and $\phi_{0}$. 2b) $a^{\prime} \neq 0, b^{\prime} \neq 0$. We then have $\chi_{0}=-b^{\prime} / a^{\prime}$. This gives $1 / 4$ BPS (F, (NS5, D5)) with $\chi_{0}=-b^{\prime} / a^{\prime}$ and arbitrary $\phi_{0}$. 
Case $\mathbf{3} \boldsymbol{a} \neq \mathbf{0}, \boldsymbol{b} \neq \mathbf{0}$. We have three subcases to consider. 3a) For $a^{\prime}=0, b^{\prime} \neq 0$, we have $\chi_{0}=a / b$ with $\phi_{0}$ arbitrary. So we have $1 / 4$ BPS threshold ((F, D1), D5) with $\chi_{0}=a / b$ and arbitrary $\phi_{0}$. 3b) $a^{\prime} \neq 0, b^{\prime}=0$. For this subcase, we have $e^{-2 \phi_{0}}=-\chi_{0}\left(\chi_{0}-a / b\right)$ with the constraint on $\chi_{0}$ as $0<\chi_{0}<a / b$ if $a b>0$ or $a / b<\chi_{0}<0$ if $a b<0$. We now have $1 / 4$ BPS ((F, D1), NS5) with the moduli $\phi_{0}$ and $\chi_{0}$ satisfying the conditions discussed. 3c) $a^{\prime} \neq 0$ and $b^{\prime} \neq 0$. This is the generic non-degenerate $1 / 4$ BPS ((F, D1), (NS5, D5)) case with its moduli $\chi_{0}$ and $\phi_{0}$ satisfying

$$
e^{-2 \phi_{0}}=-\left(\chi_{0}+\frac{b^{\prime}}{a^{\prime}}\right)\left(\chi_{0}-\frac{a}{b}\right)
$$

For this subcase, there is an interesting and very special case with $\phi_{0}=\chi_{0}=0$ for which we have $b^{\prime} a / a^{\prime} b=1$ from (3.18). Since both $\left(a^{\prime}, b^{\prime}\right)$ and $(a, b)$ are co-prime, this must imply $\left(a^{\prime}, b^{\prime}\right)=(a, b)$.

In all the above discussion, there are two additional constraints implied by (3.15) for $\chi_{0},(a, b)$ and $\left(a^{\prime}, b^{\prime}\right)$. They are

$$
a^{\prime}\left(b \chi_{0}-a\right) \leq 0, \quad b\left(a^{\prime} \chi_{0}+b^{\prime}\right)>0 \quad \text { or } \quad a^{\prime}\left(b \chi_{0}-a\right)<0, \quad b\left(a^{\prime} \chi_{0}+b^{\prime}\right) \geq 0 .
$$

For the purpose of comparison, we remark here that the 1/2 BPS ((F, D1), (NS5, D5)) puts less constraint on the moduli $\phi_{0}, \chi_{0}$ but more on the charges $(p, q)=k(a, b)$, $(m, n)=k^{\prime}(-b, a)$ with both $\left(k, k^{\prime}\right)$ and $(a, b)$ co-prime. But for the $1 / 4$ BPS ((F, D1), (NS5, D5)), on the contrary, we have more constraint on the moduli satisfying (3.18) but less on the charges $(p, q)=k(a, b),(m, n)=k^{\prime}\left(a^{\prime}, b^{\prime}\right)$ with $k k^{\prime} \neq 0$ and $(a, b)$ and $\left(a^{\prime}, b^{\prime}\right)$ being two pairs of co-prime integers.

We give below the most general $1 / 4$ BPS $\mathrm{SL}(2, \mathbb{Z})$ invariant $((\mathrm{F}, \mathrm{D} 1)$, (NS5, D5)) bound state,

$$
\begin{aligned}
d s^{2}= & \mathcal{H}_{1}^{-3 / 4} \mathcal{H}_{5}^{-1 / 4}\left(-d t^{2}+\left(d x^{5}\right)^{2}\right)+\mathcal{H}_{1}^{1 / 4} \mathcal{H}_{5}^{-1 / 4} \sum_{i=1}^{4}\left(d x^{i}\right)^{2} \\
& +\mathcal{H}_{1}^{1 / 4} \mathcal{H}_{5}^{3 / 4}\left(d r^{2}+r^{2} d \Omega_{3}^{2}\right) \\
e^{2 \phi}= & \frac{\left(\delta^{2} \mathcal{H}_{1}+\gamma^{2} \mathcal{H}_{5}\right)^{2}}{\mathcal{H}_{1} \mathcal{H}_{5}}, \quad \chi=\frac{\beta \delta \mathcal{H}_{1}+\alpha \gamma \mathcal{H}_{5}}{\delta^{2} \mathcal{H}_{1}+\gamma^{2} \mathcal{H}_{5}} \\
F_{3}^{(1)}= & -\gamma\left[\frac{2 Q_{1}}{\mathcal{H}_{1}^{2} r^{3}} d t \wedge d x^{5} \wedge d r+2 Q_{5} \epsilon_{3}\right] \\
F_{3}^{(2)}= & \alpha\left[\frac{2 Q_{1}}{\mathcal{H}_{1}^{2} r^{3}} d t \wedge d x^{5} \wedge d r+2 Q_{5} \epsilon_{3}\right]
\end{aligned}
$$

Here $\mathcal{H}_{1 / 5}=1+Q_{1 / 5} / r^{2}$ are the new harmonic functions which have the same form as the old harmonic functions but now with $Q_{1 / 5}=\Delta_{1 / 5}^{1 / 2} Q_{0}$, where $\Delta_{1 / 5}$ are as given in (3.11) and $Q_{0}=\sqrt{2} \kappa_{0} Q_{0}^{5} /\left(2 \Omega_{3}\right)=\alpha^{\prime}$ with $Q_{0}^{p}=(2 \pi)^{(7-2 p) / 2} \alpha^{\prime(3-p) / 2}[3]$. Also $\alpha, \beta, \gamma, \delta$ are the 


\begin{tabular}{|c|c|c|}
\hline & Parallel & Transverse \\
\hline $\mathrm{Dp}$ & $\mathrm{D}(\mathrm{p}-1)$ & $\mathrm{D}(\mathrm{p}+1)$ \\
\hline $\mathrm{F}$ & $\mathrm{W}$ & $\mathrm{F}$ \\
\hline $\mathrm{W}$ & $\mathrm{F}$ & $\mathrm{W}$ \\
\hline NS5 & NS5 & KK \\
\hline KK & KK & NS5 \\
\hline
\end{tabular}

Table 1. The T-duality rule along parallel or transverse direction to the branes or waves in Type II Theories.

various components of the $\mathrm{SL}(2, \mathbb{R})$ matrix (3.8) and can be read off from (3.10) as,

$$
\begin{aligned}
\alpha & =\left[q e^{-\phi_{0}}+\chi_{0}\left(q \chi_{0}-p\right) e^{\phi_{0}}\right] \Delta_{1}^{-\frac{1}{2}}=n \Delta_{5}^{-\frac{1}{2}}, \\
\beta & =p \Delta_{1}^{-\frac{1}{2}}=\left[m e^{-\phi_{0}}+\chi_{0}\left(m \chi_{0}+n\right) e^{\phi_{0}}\right] \Delta_{5}^{-\frac{1}{2}}, \\
\gamma & =\left(q \chi_{0}-p\right) e^{\phi_{0}} \Delta_{1}^{-\frac{1}{2}}=-m \Delta_{5}^{-\frac{1}{2}}, \\
\delta & =q \Delta_{1}^{-\frac{1}{2}}=\left(m \chi_{0}+n\right) e^{\phi_{0}} \Delta_{5}^{-\frac{1}{2}} .
\end{aligned}
$$

We note that since the Einstein frame metric is invariant under $\operatorname{SL}(2, \mathbb{R})$ transformation, the $\operatorname{SL}(2, \mathbb{R})$ transformed metric in $(3.20)$ has the same form as the original (D1, D5) metric given in (3.1) except that the quantity $Q_{1 / 5}$ related to the charge has been changed due to proper charge quantization. The original solution has $\chi=0$, but after $\operatorname{SL}(2, \mathbb{R})$ transformation a $\chi$ has been generated. The dilaton has also been transformed. We can easily check that asymptotically as $r \rightarrow \infty, \mathcal{H}_{1 / 5} \rightarrow 1$ and therefore $\phi \rightarrow \phi_{0}$ and $\chi \rightarrow \chi_{0}$ as expected. We also note that there is no NSNS 3-form in the initial (D1, D5) solution, but it has been generated by $\operatorname{SL}(2, \mathbb{R})$ transformation. However, no 5 -form field has been generated as opposed to the $1 / 2$ BPS ((F, D1), (NS5, D5)) state [3].

Following the steps given in [8] and [9], the string-frame tension of the bound state $((\mathrm{F}, \mathrm{D} 1),(\mathrm{NS} 5, \mathrm{D} 5))$ can be computed from the ADM mass per unit 5-brane volume as

$$
T_{5}\left(k, k^{\prime} ; a, b ; a^{\prime}, b^{\prime}\right)=\frac{T_{0}^{5}}{g}\left(k \sqrt{b^{2}+\left(b \chi_{0}-a\right)^{2} g^{2}}+k^{\prime} \sqrt{a^{\prime 2} g^{-2}+\left(a^{\prime} \chi_{0}+b^{\prime}\right)^{2}}\right),
$$

where $T_{0}^{p}=1 /\left[(2 \pi)^{p} \alpha^{\prime(p+1) / 2}\right]$ is the $p$-brane tension unit and $g=e^{\phi_{0}}$ is the string coupling. Here we have also set $(p, q)=k(a, b),(m, n)=k^{\prime}\left(a^{\prime}, b^{\prime}\right)$ with $(a, b)$ and $\left(a^{\prime}, b^{\prime}\right)$ both co-prime. This tension is the sum of contributions from $(p, q)$ strings and $(m, n) 5$-branes, respectively, and this is actually determined by the form of the metric given in (3.20), which is the same in form as that of (D1, D5), mentioned earlier. This tension gives all expected properties, for example, the dependence of string coupling for each kind of constituent branes. We will not discuss them explicitly here.

One can also get various 1/4 BPS descendant states from this one using T-dualities along the 5-brane worldvolume isometric directions or directions transverse to the 5-branes following the standard rule as given in table 1. 
In the above table, KK and $\mathrm{W}$ stand for KK-monopole and waves, respectively. So, for example, if we take a T-duality transformation along the string direction, we will end up with a $1 / 4$ BPS $((\mathrm{W}, \mathrm{D} 0),(\mathrm{NS} 5, \mathrm{D} 4))$ and if we take T-duality along the 5-brane but not the string direction, we end up with a 1/4 BPS ((F, D2), (NS5, D4)) and so on. We can also take T-duality along one of transverse directions to the 5-branes for which we first need have one isometry along this T-dual direction. We then end up with $1 / 4$ BPS ((F, D2), (KK, D6)).

\section{No-force condition}

As discussed in the Introduction, we, in general, expect an attractive interaction between the non-threshold $(p, q)$ strings and the non-threshold $(m, n) 5$-branes due to the attractive force between F and D5 as well as between D1 and NS5. If this interaction cannot be turned off, the 1/4 BPS threshold ((F, D1), (NS5, D5)) state cannot exist. However, in the previous section, using the classical $\mathrm{SL}(2, \mathbb{R})$ transformation on a $1 / 4$ BPS (D1, D5) state and then imposing the charge quantization for each kind of constituent branes, we seem to have obtained such $1 / 4$ BPS threshold state when the moduli $\phi_{0}$ and $\chi_{0}$ satisfy the condition (3.12) or (3.14) or (3.18). This, therefore, suggests that the condition on the moduli just mentioned, actually plays a key role to change the nature of force, contrary to our naive understanding, such that the net force acting between (F, D1) and (NS5, D5) vanishes. Otherwise, what we have found in the previous section is simply wrong. Therefore, to cross-check our finding on the 1/4 BPS threshold ((F, D1), (NS5, D5)) state, we need to explicitly compute the net force to see whether it can vanish and under what condition. We find nicely that the condition for which the net force vanishes is precisely the one given in the previous section for the moduli $\phi_{0}$ and $\chi_{0}$.

For this purpose, let us place a $(p, q)$ string in an $(m, n) 5$-brane background and see, under what condition the force between the two vanishes. The $(m, n) 5$-brane background [2] is

$$
\begin{aligned}
d s^{2} & =A_{(m, n)}^{1 / 4}\left(-d t^{2}+d x^{i} d x^{j} \delta_{i j}\right)+A_{(m, n)}^{-3 / 4}\left(d r^{2}+r^{2} d \Omega_{3}^{2}\right), \\
e^{-\phi} & =\frac{\Delta_{5} A_{(m, n)}^{1 / 2} e^{\phi_{0}}}{m^{2} e^{-\phi_{0}}+A_{(m, n)} e^{\phi_{0}}\left(m \chi_{0}+n\right)^{2}}, \\
\chi & =\frac{\chi_{0} \Delta_{5} A_{(m, n)}+m n e^{-\phi_{0}}\left(A_{(m, n)}-1\right)}{m^{2} e^{-\phi_{0}}+A_{(m, n)} e^{\phi_{0}}\left(m \chi_{0}+n\right)^{2}}, \\
F_{3}^{(1)} & =2 m Q_{0} \epsilon_{3}, \quad F_{3}^{(2)}=2 n Q_{0} \epsilon_{3},
\end{aligned}
$$

where the metric is in the Einstein frame and in the above

$$
A_{(m, n)}^{-1}=1+\frac{Q_{(m, n)}}{r^{2}}, \text { with } Q_{(m, n)}=\Delta_{5}^{1 / 2} Q_{0},
$$

with $\Delta_{5}$ defined earlier for $(m, n) 5$-brane in (3.11) and $Q_{0}=\sqrt{2} \kappa_{0} Q_{0}^{5} /\left(2 \Omega_{3}\right)$ (Note here $\left.Q_{0}^{p}=(2 \pi)^{(7-2 p) / 2} \alpha^{\prime(3-p) / 2}\right)$. There are various forms of $(p, q)$ string action [10-12] and for convenience we here use the bosonic part of $(p, q)$ string action which was first given 
in [13]. Since $(p, q)$ string will not couple to $(m, n)$ 5-brane magnetic 3 -form field strength, we only need to consider the part of the couplings of $(p, q)$ string with the background metric, dilaton and the 0 -form $\chi$ of the $(m, n) 5$-brane. It is given as

$$
\begin{aligned}
S & =-T \int d^{2} \sigma \sqrt{q^{2} e^{-\phi}+(p-q \chi)^{2} e^{\phi}} \sqrt{-h} \\
& =-T \int d^{2} \sigma \bar{\Delta}_{1}^{1 / 2} \sqrt{-h},
\end{aligned}
$$

where

$$
\bar{\Delta}_{1}=(p, q)^{T} \mathcal{M}^{-1}\left(\begin{array}{c}
p \\
q
\end{array}\right)=q^{2} e^{-\phi}+(p-q \chi)^{2} e^{\phi} .
$$

Here $\mathcal{M}$ is the scalar matrix defined in (2.4), and $h=\operatorname{det} h_{\alpha \beta}$, with $h_{\alpha \beta}$, the induced world-sheet metric from spacetime Einstein metric $g_{M N}$ given in (4.1) as

$$
h_{\alpha \beta}=\partial_{\alpha} X^{M} \partial_{\beta} X^{N} g_{M N},
$$

where the worldsheet indices $\alpha, \beta=0,1$. So from the second line of the action (4.3), it is clear that the action is manifestly $\operatorname{SL}(2, \mathbb{Z})$ invariant. The equation of motion for $X^{M}$ can be derived from the action (4.3) and after a lengthy calculation we obtain it as

$$
\frac{1}{\sqrt{-h}} \partial_{\alpha}\left(\sqrt{-h} h^{\alpha \beta} \partial_{\beta} X^{M}\right)+V^{M}=0,
$$

where

$$
\begin{aligned}
V^{M}= & \Gamma_{N P}^{M} h^{\alpha \beta} \partial_{\alpha} X^{N} \partial_{\beta} X^{P} \\
& +\frac{2 q(q \chi-p) \partial_{N} \chi+\left((q \chi-p)^{2}-q^{2} e^{-2 \phi}\right) \partial_{N} \phi}{2 \bar{\Delta}_{1} e^{-\phi}}\left(h^{\alpha \beta} \partial_{\alpha} X^{N} \partial_{\beta} X^{M}-g^{N M}\right) .
\end{aligned}
$$

In the above,

$$
\Gamma_{N P}^{M}=\frac{1}{2} g^{M Q}\left(\partial_{N} g_{Q P}+\partial_{P} g_{Q N}-\partial_{Q} g_{N P}\right),
$$

is the usual Christoffel symbol. We now look for the conditions for which the force $V^{M}$ between $(p, q)$ string and $(m, n)$ 5-brane vanishes when $(p, q)$ string is placed along one of the five spatial directions $\left(x^{1}, x^{2}, \cdots, x^{5}\right.$, say $\left.x^{1}\right)$ of the 5 -brane. In other words, we are looking for the "no-force" condition. We also take the so-called static gauge for the string, namely, $\tau=X^{0}, \sigma=X^{1}$ with string worldsheet coordinates $\sigma^{\alpha}=(\tau, \sigma)$. If string is parallel to 5 -brane and is static, vanishing force, i.e., $V^{M}=0$, would then imply from (4.6) that it will remain static. So, for now, all the $X^{M}$ 's except $X^{0}=\tau, X^{1}=\sigma$ will be independent of $\tau, \sigma$. We then have

$$
h_{\alpha \beta}=\partial_{\alpha} X^{M} \partial_{\beta} X^{N} g_{M N}=g_{11} \eta_{\alpha \beta},
$$

where $g_{11}=A_{(m, n)}^{1 / 4}$ as given by the metric (4.1) and $\eta_{\alpha \beta}=(-1,1)$ the worldsheet flat metric. The relevant $\Gamma_{N P}^{M}$ for $V^{M}$ are $\Gamma_{00}^{M}$ and $\Gamma_{11}^{M}$ and their non-vanishing components are

$$
\Gamma_{00}^{r}=-\Gamma_{11}^{r}=\frac{1}{2} g^{r r} \partial_{r} g_{11} .
$$


Note that both $\chi$ and $\phi$ are only functions of $r$ and so automatically $V^{M}=0$ for all $M$ except for $M=r$. Now we need to check under what condition $V^{r}=0$. The first term on the right hand side of (4.7) can be expressed as

$$
\Gamma_{N P}^{M} h^{\alpha \beta} \partial_{\alpha} X^{N} \partial_{\beta} X^{P}=-g^{r r} g^{11} \partial_{r} g_{11}=-\frac{1}{4} g^{r r} A_{(m, n)}^{-1} \partial_{r} A_{(m, n)},
$$

where in the last equality the metric component $g_{11}$ given in (4.1) has been used. From $\chi$ and $\phi$ given in (4.1), we also have

$$
\begin{aligned}
\partial_{r} \chi & =\frac{m\left(m \chi_{0}+n\right) e^{-\phi_{0}} \Delta_{5} \partial_{r} A_{(m, n)}}{\left[m^{2} e^{-\phi_{0}}+A_{(m, n)}\left(m \chi_{0}+n\right)^{2} e_{0}^{\phi}\right]^{2}}, \\
\partial_{r} \phi & =\frac{\left[A_{(m, n)}\left(m \chi_{0}+n\right)^{2}-m^{2} e^{-2 \phi_{0}}\right] e^{\phi_{0}}}{2\left[m^{2} e^{-\phi_{0}}+A_{(m, n)}\left(m \chi_{0}+n\right)^{2} e_{0}^{\phi}\right]} A_{(m, n)}^{-1} \partial_{r} A_{(m, n)} .
\end{aligned}
$$

We then have from (4.7),

$$
-V_{r}=\frac{1}{4} A_{(m, n)}^{-1} \partial_{r} A_{(m, n)}+\frac{1}{2} \frac{2 q(q \chi-p) \partial_{r} \chi+\left((q \chi-p)^{2}-q^{2} e^{-2 \phi}\right) \partial_{r} \phi}{2\left[(q \chi-p)^{2}+q^{2} e^{-2 \phi}\right]},
$$

where $V_{r} \equiv g_{r r} V^{r}$ and we have used $\bar{\Delta}_{1}$ given in (4.4). At first look, the simplification of the right hand side of (4.13) might appear difficult. But a careful examination reveals that a great simplification can be achieved if we first combine the first term on the right hand side of (4.13) with the term involving $\partial_{r} \phi$ and then we add the term involving $\partial_{r} \chi$. Let us see this in a bit detail. The first combination just described gives

$$
\begin{aligned}
& \frac{\partial_{r} A_{(m, n)} e^{\phi_{0}}}{2 \bar{\Delta}_{1} e^{-\phi}\left[m^{2} e^{-\phi_{0}}+A_{(m, n)}\left(m \chi_{0}+n\right)^{2} e^{\phi_{0}}\right]^{3}}\left\{q^{2} m^{2} \Delta_{5}^{2} e^{-4 \phi_{0}}\right. \\
& \left.+\left(m \chi_{0}+n\right)^{2}\left[\left(q \Delta_{5} \chi_{0}+q m n e^{-\phi_{0}}-p\left(m \chi_{0}+n\right)^{2} e^{\phi_{0}}\right) A_{(m, n)}-m(q n+p m) e^{-\phi_{0}}\right]^{2}\right\},
\end{aligned}
$$

where $\partial_{r} \phi$ given in (4.12) has been used. The term involving $\partial_{r} \chi$ on the right hand side of (4.13) can be expressed as

$$
\begin{aligned}
\frac{q(q \chi-p) \partial_{r} \chi}{2 \bar{\Delta}_{1} e^{-\phi}}= & \frac{q m\left(m \chi_{0}+n\right) \Delta_{5} e^{-\phi_{0}} \partial_{r} A_{(m, n)}}{2 \bar{\Delta}_{1} e^{-\phi}\left[m^{2} e^{-\phi_{0}}+A_{(m, n)}\left(m \chi_{0}+n\right)^{2} e^{\phi_{0}}\right]^{3}} \\
& \times\left[\left(q \Delta_{5} \chi_{0}+q m n e^{-\phi_{0}}-p\left(m \chi_{0}+n\right)^{2} e^{\phi_{0}}\right) A_{(m, n)}-m(q n+p m) e^{-\phi_{0}}\right] .
\end{aligned}
$$

Combining (4.14) with (4.15), we end up with

$$
V_{r}=-\frac{\partial_{r} A_{(m, n)} e^{\phi_{0}}}{2 \bar{\Delta}_{1} e^{-\phi}\left[m^{2} e^{-\phi_{0}}+A_{(m, n)}\left(m \chi_{0}+n\right)^{2} e^{\phi_{0}}\right]^{3}} \bar{V}_{r},
$$

where

$$
\begin{aligned}
\bar{V}_{r}=\{ & \left(m \chi_{0}+n\right)\left[\left(q \Delta_{5} \chi_{0}+q m n e^{-\phi_{0}}-p\left(m \chi_{0}+n\right)^{2} e^{\phi_{0}}\right) A_{(m, n)}-m(q n+p m) e^{-\phi_{0}}\right] \\
& \left.+q m \Delta_{5} e^{-2 \phi_{0}}\right\}^{2}
\end{aligned}
$$


is a perfect square. So $V_{r}=0$ amounts to setting $\bar{V}_{r}=0$, which implies

$$
\begin{aligned}
\left(m \chi_{0}+n\right)\left[q \Delta_{5} \chi_{0}+q m n e^{-\phi_{0}}-p\left(m \chi_{0}+n\right)^{2} e^{\phi_{0}}\right] & =0, \\
q m \Delta_{5} e^{-2 \phi_{0}}-m\left(m \chi_{0}+n\right)(q n+m p) e^{-\phi_{0}} & =0 .
\end{aligned}
$$

The above two equations can be further simplified to give

$$
\begin{aligned}
\left(m \chi_{0}+n\right)^{2}\left[q m e^{-2 \phi_{0}}+\left(m \chi_{0}+n\right)\left(q \chi_{0}-p\right)\right] & =0, \\
m^{2}\left[q m e^{-2 \phi_{0}}+\left(m \chi_{0}+n\right)\left(q \chi_{0}-p\right)\right] & =0 .
\end{aligned}
$$

These two equations amount to requiring

$$
q m e^{-2 \phi_{0}}+\left(m \chi_{0}+n\right)\left(q \chi_{0}-p\right)=0,
$$

which is nothing but the condition (3.12) derived in the previous section for the existence of 1/4 BPS ((F, D1), (NS5, D5)) state. We can also set $(p, q)=k(a, b),(m, n)=k^{\prime}\left(a^{\prime}, b^{\prime}\right)$ with again $(a, b)$ and $\left(a^{\prime}, b^{\prime}\right)$ being two pairs of co-prime integers. Here again the generic consideration of the force between $(\mathrm{F}, \mathrm{D} 1)$ and $(\mathrm{NS} 5, \mathrm{D} 5)$ requires $k k^{\prime} \neq 0$. Then the discussion of the "no-force" condition will go exactly the same way as that for the existence of $1 / 4$ BPS state given in the previous section and so we will not repeat it here.

So we conclude that when a $(p, q)$ string is placed in an $(m, n) 5$-brane background with a given vacuum, i.e., with given $\phi_{0}$ and $\chi_{0}$, along one of the 5 -brane world-volume spatial directions, one in general expects a net attractive force between them since we know that in general the forces between the constituent branes are either attractive or zero (the force between F and D5 as well as between D1 and NSNS5 is attractive while the others are zero) and therefore the end result is a non-threshold 1/2 BPS ((F, D1), (NS5, D5)) state as discussed in [3]. However, when the no-force condition (4.20) holds, then the end result is a $1 / 4$ BPS threshold bound state ((F, D1), (NS5, D5)) as discussed in this paper.

\section{Another 1/4 BPS ((F, D1), (NS5, D5)) from 1/4 BPS (F, D5)}

In this section, we will give the construction of another 1/4 BPS ((F, D1), (NS5, D5)) state starting from a known 1/4 BPS (F, D5) state of type IIB string theory for completeness. Unlike in the previous case here $(p, q)$ string is perpendicular to the $(m, n) 5$-brane. However, the construction and the charge relation in this case are very similar to those of $1 / 2$ BPS ((F, D1), (NS5, D5)) state we constructed earlier in [3] and so we will be brief here. The 1/4 BPS (F, D $p$ ) threshold bound states were constructed in [14], so, we write down the (F, D5) solution from that reference, putting $p=5$ in their eq. (2.6), in the following,

$$
\begin{aligned}
d s^{2}= & -H_{1}^{-3 / 4} H_{5}^{-1 / 4} d t^{2}+H_{1}^{1 / 4} H_{5}^{-1 / 4} \sum_{i=1}^{5}\left(d x^{i}\right)^{2} \\
& +H_{1}^{-3 / 4} H_{5}^{3 / 4}\left(d x^{6}\right)^{2}+H_{1}^{1 / 4} H_{5}^{3 / 4}\left(d r^{2}+r^{2} d \Omega_{2}^{2}\right), \\
e^{2 \phi}= & \frac{1}{H_{1} H_{5}}, \\
F_{3}^{(1)}= & \frac{Q_{1}}{H_{1}^{2} r^{2}} d t \wedge d x^{6} \wedge d r, \\
F_{3}^{(2)}= & Q_{5} \epsilon_{2} \wedge d x^{6} .
\end{aligned}
$$


Note that in the above we have written the metric in the Einstein frame. From the form of the metric it is clear that the $\mathrm{F}$-string is along $x^{6}$ directions and delocalized in $x^{1}, x^{2}, \ldots, x^{5}$ directions whereas, the D5 brane is lying along $x^{1}, x^{2}, \ldots, x^{5}$ directions and delocalized in $x^{6}$ direction. $F_{3}^{(1)}$ and $F_{3}^{(2)}$ are the NSNS and RR 3-forms respectively. Again in the above solution we have set the asymptotic values $\phi_{0}=0$ and $\chi_{0}=0$. The harmonic functions now have the forms $H_{1 / 5}=1+Q_{1 / 5} / r$, where $Q_{1 / 5}$ are related to F-string and D5-brane charges as,

$$
e_{1}=\frac{Q_{1} \Omega_{2} V_{5}}{\sqrt{2} \kappa_{0}}, \quad g_{5}=\frac{Q_{5} \Omega_{2} V_{1}}{\sqrt{2} \kappa_{0}} .
$$

Here $\Omega_{2}=4 \pi$ is the area of the unit two sphere and $V_{1}=\int d x^{6}, V_{5}=\int d x^{1} \wedge \ldots \wedge d x^{5}$. The electric-like (F, D1) string charge $e_{1}^{(i)}$ and the magnetic-like (NS5, D5) brane charge $g_{5}^{(i)}$ are given in (3.5) and (3.6) respectively, however unlike in that case the integration region in this case should be $R^{5} \times S_{\infty}^{2}$ for the electric-like charge and $R^{1} \times S_{\infty}^{2}$ for the magnetic-like charge. As before, the electric-like and magnetic-like charges transform under $\operatorname{SL}(2, \mathbb{R})$ as $e_{1} \rightarrow \Lambda e_{1}$ and $g_{5} \rightarrow\left(\Lambda^{-1}\right)^{T} g_{5}$, with the standard choice of $\operatorname{SL}(2, \mathbb{R})$ matrix as given in (3.8). Since our initial classical configuration (F, D5) has classical F-string charge $\bar{\Delta}_{1}^{1 / 2}$ and classical D5-brane charge $\bar{\Delta}_{5}^{1 / 2}$, we have

$$
e_{1}=\left(\begin{array}{c}
\bar{\Delta}_{1}^{1 / 2} \\
0
\end{array}\right) e_{10}, \quad g_{5}=\left(\begin{array}{c}
0 \\
\bar{\Delta}_{5}^{1 / 2}
\end{array}\right) g_{50},
$$

where as usual $e_{10}$ and $g_{50}$ are the unit charges of the strings and the 5 -branes. the $\mathrm{SL}(2, \mathbb{R})$ transformed charges after imposing the quantization would be given as, $\bar{e}_{1}^{T}=(p, q) e_{10}$ and $\bar{g}_{5}^{T}=(m, n) g_{50}$, where $(p, q)$ and $(m, n)$ are two pairs of integers. These quantization conditions give us the following relations

$$
\begin{aligned}
& \cos \alpha=-\left(q \chi_{0}-p\right) e^{\phi_{0} / 2} \bar{\Delta}_{1}^{-1 / 2}=\left(m \chi_{0}+n\right) e^{\phi_{0} / 2} \bar{\Delta}_{5}^{-1 / 2}, \\
& \sin \alpha=q e^{-\phi_{0} / 2} \bar{\Delta}_{1}^{-1 / 2}=-m e^{-\phi_{0} / 2} \bar{\Delta}_{5}^{-1 / 2},
\end{aligned}
$$

and using these relations we determine

$$
\begin{aligned}
\bar{\Delta}_{1} & =q^{2} e^{-\phi_{0}}+\left(q \chi_{0}-p\right)^{2} e^{\phi_{0}}, \quad \bar{\Delta}_{5}=m^{2} e^{-\phi_{0}}+\left(m \chi_{0}+n\right)^{2} e^{\phi_{0}}, \\
\tan \alpha & =\frac{-q e^{-\phi_{0}}}{q \chi_{0}-p}=\frac{-m e^{-\phi_{0}}}{m \chi_{0}+n} .
\end{aligned}
$$

The last relation can be satisfied if $p m=-q n$. This in turn implies that the integers must have the forms $(p, q)=k(a, b)$ and $(m, n)=k^{\prime}(-b, a)$, where $(a, b)$ and $\left(k, k^{\prime}\right)$ are two pairs of co-prime integers. Note that here the moduli $\phi_{0}$ and $\chi_{0}$ can be arbitrary unlike in the previous case. So this state is very similar to the $1 / 2$ BPS ((F, D1), (NS5, D5)) one. The discussion of how to recover all the special case states from the general 1/4 BPS ((F, D1), (NS5, D5)) state in this case is also very similar to that given in [3] and therefore will not be repeated here. The complete $\mathrm{SL}(2, \mathbb{Z})$ invariant $1 / 4$ BPS ((F, D1), (NS5, D5)) threshold 
bound state solution where strings are perpendicular to the 5-branes is given below,

$$
\begin{aligned}
d s^{2}= & -\mathcal{H}_{1}^{-3 / 4} \mathcal{H}_{5}^{-1 / 4} d t^{2}+\mathcal{H}_{1}^{1 / 4} \mathcal{H}_{5}^{-1 / 4} \sum_{i=1}^{5}\left(d x^{i}\right)^{2} \\
& +\mathcal{H}_{1}^{-3 / 4} \mathcal{H}_{5}^{3 / 4}\left(d x^{6}\right)^{2}+\mathcal{H}_{1}^{1 / 4} \mathcal{H}_{5}^{3 / 4}\left(d r^{2}+r^{2} d \Omega_{2}^{2}\right), \\
e^{2 \phi}= & \frac{\left(\gamma^{2} \mathcal{H}_{1} \mathcal{H}_{5}+\delta^{2}\right)^{2}}{\mathcal{H}_{1} \mathcal{H}_{5}}, \quad \chi=\frac{\alpha \gamma \mathcal{H}_{1} \mathcal{H}_{5}+\beta \delta}{\gamma^{2} \mathcal{H}_{1} \mathcal{H}_{5}+\delta^{2}}, \\
F_{3}^{(1)}= & \delta \frac{Q_{1}}{\mathcal{H}_{1}^{2} r^{2}} d t \wedge d x^{6} \wedge d r-\gamma Q_{5} \epsilon_{2} \wedge d x^{6} \\
F_{3}^{(2)}= & -\beta \frac{Q_{1}}{\mathcal{H}_{1}^{2} r^{2}} d t \wedge d x^{6} \wedge d r+\alpha Q_{5} \epsilon_{2} \wedge d x^{6},
\end{aligned}
$$

where $\mathcal{H}_{1 / 5}=1+Q_{1 / 5} / r$ are the new harmonic functions with the same form as the old ones, but $Q_{1 / 5}$ have now changed to $\bar{\Delta}_{1 / 5}^{1 / 2} Q_{0}$. Here $\bar{\Delta}_{1 / 5}$ are as given in (5.5) and $Q_{0}=\sqrt{2} \kappa_{0} Q_{0}^{5} /\left(\omega_{2} V_{1}\right)$, with $Q_{0}^{5}$ as defined before. The $\operatorname{SL}(2, \mathbb{R})$ parameters $\alpha, \beta, \gamma, \delta$ can be obtained from (3.8) with the use of (5.4) and are given as,

$$
\begin{aligned}
& \alpha=p \bar{\Delta}_{1}^{-\frac{1}{2}}=n \bar{\Delta}_{5}^{-\frac{1}{2}}, \\
& \beta=-\left[q e^{-\phi_{0}}+\chi_{0}\left(q \chi_{0}-p\right) e^{\phi_{0}}\right] \bar{\Delta}_{1}^{-\frac{1}{2}}=\left[m e^{-\phi_{0}}+\chi_{0}\left(m \chi_{0}+n\right) e^{\phi_{0}}\right] \bar{\Delta}_{5}^{-\frac{1}{2}}, \\
& \gamma=q \bar{\Delta}_{1}^{-\frac{1}{2}}=-m \bar{\Delta}_{5}^{-\frac{1}{2}} \\
& \delta=-\left(q \chi_{0}-p\right) e^{\phi_{0}} \bar{\Delta}_{1}^{-\frac{1}{2}}=\left(m \chi_{0}+n\right) e^{\phi_{0}} \bar{\Delta}_{5}^{-\frac{1}{2}} .
\end{aligned}
$$

As before we notice here that the $\mathrm{SL}(2, \mathbb{R})$ transformed metric in (5.6) retains its form as the original metric given in (5.1) (as the metric is given in the Einstein frame) except for $Q_{1 / 5}$ which have been changed due to the corresponding charge quantizations, respectively. But the dilaton has been changed and a $\chi$ has been generated due to the $\operatorname{SL}(2, \mathbb{R})$ transformation even though the original solution does not contain a $\chi$. The 3 -form fields have also been changed but no 5 -form field has been generated as in the previous case and unlike the $1 / 2$ BPS case. Again it can be easily checked that as $r \rightarrow \infty, \mathcal{H}_{1 / 5} \rightarrow 1$ and therefore, $\phi \rightarrow \phi_{0}$ and $\chi \rightarrow \chi_{0}$ as expected.

One can calculate the string frame tension of this bound state from the ADM mass per unit 5-brane volume following [8] and [9] and we get,

$$
T_{5}\left(k, k^{\prime} ; a, b\right)=\frac{T_{0}^{5}}{g}\left(k+\frac{k^{\prime}}{g}\right) \sqrt{b^{2}+\left(b \chi_{0}-a\right)^{2} g^{2}}
$$

where $T_{0}^{5}$ is as defined before and $g=e^{\phi_{0}}$ is the string coupling. This tension is the sum of the tensions of $(p, q)$ strings and $(m, n) 5$-branes. One can easily check that this expression gives all the expected tensions of the constituent branes.

Using the T-duality rule given in table 1 one can generate various $1 / 4$ BPS descendant states, by applying T-duality, starting from this $1 / 4$ BPS ((F, D1), (NS5, D5)) state. So, for example, applying T-duality along $x^{6}$, we can get $1 / 4$ BPS ((W, D0), (KK, D6)) state 
and applying T-duality along $x^{5}$, we can get $1 / 4$ BPS ((F, D2), (NS5, D4)) and again applying T-duality along $x^{4}$ on this state we can get 1/4 BPS ((F, D3), (NS5, D3)) state and so on. All these states are asymptotically flat and other such asymptotically flat states cannot be generated by applying T-duality along the directions transverse to both strings and 5-branes in this case.

\section{Conclusion}

On one hand it is known that when $(\mathrm{F}, \mathrm{D} 1)$ or $(p, q)$ strings are placed parallel to one of the world-volume spatial directions of (NS5, D5) or $(m, n) 5$-branes, they should attract each other since F-strings attract D5 branes and D1-strings attract NS5 branes and therefore, when they form bound state, it should only be 1/2 BPS ((F, D1), (NS5, D5)) state. On the other hand, we know that type IIB string theory admits 1/4 BPS (D1, D5) bound state solution and so by applying the $\operatorname{SL}(2, \mathbb{R})$ classical symmetry of type IIB string theory and imposing charge quantization we should be able to construct an $\operatorname{SL}(2, \mathbb{Z})$ invariant $1 / 4$ BPS $((\mathrm{F}, \mathrm{D} 1),(\mathrm{NS} 5, \mathrm{D} 5))$ bound state in this theory where $(p, q)$ strings and $(m, n) 5$-branes should not feel any attractive force on each other. In this paper we have resolved this apparent contradiction by explicitly constructing this new vacua, i.e., 1/4 BPS ((F, D1), (NS5, D5)) state of type IIB string theory from 1/4 BPS (D1, D5) state and applying $\mathrm{SL}(2, \mathbb{R})$ symmetry as well as the charge quantization condition to it. We found that the consistency condition for the existence of this bound state manifests itself in the form of certain constraint given by (3.12) (or (3.14) or (3.18)) on the vaccum moduli of the solution. We have discussed and pointed out many subtleties on how to recover the special case bound states from this general bound state solution not faced before in similar constructions. We then computed the force experienced by a probe $(\mathrm{F}, \mathrm{D} 1)$ or $(p, q)$ string placed in the background of (NS5, D5) or $(m, n) 5$-branes. We found that when the string is parallel to one of the spatial world-volume directions of the 5-brane, the force can vanish under certain condition. Nicely, we found that the no-force condition precisely matches with the condition on the moduli (3.12) we obtained before while constructing the $\mathrm{SL}(2, \mathbb{Z})$ invariant $1 / 4$ BPS ((F, D1), (NS5, D5)) state. Further we have given the tension formula for this general bound state from the ADM mass and also discussed how to obtain various other descendant $1 / 4$ BPS bound states from this by the application of T-dualities in various isometric directions.

For completeness, we have also constructed another $\mathrm{SL}(2, \mathbb{Z})$ invariant new vacua of type IIB string theory in the form of a $1 / 4$ BPS ((F, D1), (NS5, D5)) bound state where, strings are perpendicular to 5-branes. In this case we started from the known 1/4 BPS (F, D5) bound state solution of type IIB theory and applied $\mathrm{SL}(2, \mathbb{R})$ transformation as well as the charge quantization condition to it. We pointed out that the construction of this state is very similar to that of the $1 / 2$ BPS ((F, D1), (NS5, D5)) state, constructed before by two of us in [3]. In this case there is no extra condition on the vacuum moduli unlike in the previous case and they can be completely arbitrary. Here also we have given the tension formula and discussed how the other descendant 1/4 BPS bound states can be obtained by T-duality. 


\section{Acknowledgments}

We would like to thank our anonymous referees for fruitful suggestions which help us to improve the manuscript and also for bringing reference 13 to our attention. QJ and JXL acknowledge support by a key grant from the NSF of China with Grant No: 11235010.

Open Access. This article is distributed under the terms of the Creative Commons Attribution License (CC-BY 4.0), which permits any use, distribution and reproduction in any medium, provided the original author(s) and source are credited.

\section{References}

[1] J.H. Schwarz, An $\mathrm{SL}(2, \mathbb{Z})$ multiplet of type IIB superstrings, Phys. Lett. B 360 (1995) 13 [Erratum ibid. B 364 (1995) 252] [hep-th/9508143] [INSPIRE].

[2] J.X. Lu and S. Roy, An SL(2, Z) multiplet of type IIB super five-branes, Phys. Lett. B 428 (1998) 289 [hep-th/9802080] [INSPIRE].

[3] J.X. Lu and S. Roy, (F, D5) bound state, $\mathrm{SL}(2, \mathbb{Z})$ invariance and the descendant states in type IIB/A string theory, Phys. Rev. D 60 (1999) 126002 [hep-th/9905056] [INSPIRE].

[4] J. Ouyang and C. Wu, A classification of long-range interactions between two stacks of $p \&$ p'-branes, Commun. Theor. Phys. 63 (2015) 195 [arXiv:1409.0969] [INSPIRE].

[5] J.H. Schwarz, Covariant Field Equations of Chiral $N=2$ D $=10$ Supergravity, Nucl. Phys. B 226 (1983) 269 [INSPIRE].

[6] H. Lü, C.N. Pope and T.A. Tran, Five-dimensional $N=4$, $\mathrm{SU}(2) \times \mathrm{U}(1)$ gauged supergravity from type IIB, Phys. Lett. B 475 (2000) 261 [hep-th/9909203] [INSPIRE].

[7] J.X. Lu, S. Roy, Z.-L. Wang and R.-J. Wu, Non-supersymmetric D1/D5, F/NS5 and closed string tachyon condensation, Nucl. Phys. B 819 (2009) 282 [arXiv:0903.3310] [INSPIRE].

[8] J.X. Lu and S. Roy, Nonthreshold (F, Dp) bound states, Nucl. Phys. B 560 (1999) 181 [hep-th/9904129] [INSPIRE].

[9] J.X. Lu, ADM masses for black strings and p-branes, Phys. Lett. B 313 (1993) 29 [hep-th/9304159] [INSPIRE].

[10] P.K. Townsend, Membrane tension and manifest IIB S duality, Phys. Lett. B 409 (1997) 131 [hep-th/9705160] [INSPIRE].

[11] M. Cederwall and P.K. Townsend, The Manifestly $\mathrm{SL}(2, \mathbb{Z})$ covariant superstring, JHEP 09 (1997) 003 [hep-th/9709002] [inSPIRE].

[12] E. Bergshoeff, P.S. Howe, S. Kerstan and L. Wulff, Kappa-symmetric $\operatorname{SL}(2, \mathbb{R})$ covariant D-brane actions, JHEP 10 (2007) 050 [arXiv: 0708.2722] [INSPIRE].

[13] E.A. Bergshoeff, M. de Roo, S.F. Kerstan, T. Ortín and F. Riccioni, SL(2, R)-invariant IIB Brane Actions, JHEP 02 (2007) 007 [hep-th/0611036] [INSPIRE].

[14] P. Dey and S. Roy, Lifshitz-like space-time from intersecting branes in string/M theory, JHEP 06 (2012) 129 [arXiv: 1203.5381] [INSPIRE]. 